Proc. XIX International School of Semiconducting Compounds, Jaszowiec 1990

\title{
RECONSTRUCTION OF THE (001) SURFACE OF Si FROM MOLECULAR DYNAMICS*
}

\author{
J.M. Holender AND CZ. JęDRZEJEK \\ Departament of Physics, Jagellonian University, ul. Reymonta 4, 30-059 Kraków, Poland
}

(Received August 8, 1990)

\begin{abstract}
The reconstruction of the (001) surface of $\mathrm{Si}$ at various temperatures is studied using molecular dynamics with many-body interactions. Two types of potentials were used: the Stillinger-Weber (SW), and Pearson and co-workers Axilrod-Teller type potential (AT). For Stillinger-Weber potential at low temperatures the $(2 \times 1)$ dimer reconstruction is about $0.07 \mathrm{eV}$ per surface atom more preferable than the $c(2 \times 2)$ structure which is in agreement with the experimental reconstruction observed by STM. Contrary, for Axilrod-Teller type potential the $c(2 \times 2)$ structure is lower by $0.2 \mathrm{eV}$ than the $(2 \times 1)$ structure. The silicon surface is stable up to $1500 \mathrm{~K}$, all the dimers remain unbroken but some of them are tilted. The energies of various defects (suggested by STM studies) like single vacancy, two adjacent Si atoms vacancy, dimer vacancy, dimer vacancy with lower layer atoms rebonding and double dimer vacancy are estimated.
\end{abstract}

PACS numbers: 68.35.Bs, 68.35.Md

The reconstruction of the (001) surface of Si is studied using NVT molecular dynamics (MD) technique. From the variety of model potentials [1-6] two types have been chosen for this study: the Stillinger-Weber (SW), [1] and Pearson and co-workers Axilrod-Teller type potential (AT) [2].The first is chosen because of its simplicity, and the second is applied because this potential have been derived for the $\mathrm{Ga}$-As system as well [7] so that the MD simulation of $\mathrm{Ga}-\mathrm{As}$ epitaxial growth on $\mathrm{Si}$ would be possible using this potential.

Used potentials (AT and SW) compose of two and three body parts. The pair potentials are insufficient for making diamond structure stable, so that at least three body contributions are necessary. The total energy of system $U$ can be expressed as:

$$
U=\frac{1}{2} \sum_{i} \sum_{j \neq i} U_{2}\left(r_{i}, r_{j}, r_{k}\right)+\frac{1}{6} \sum_{i} \sum_{j \neq i} \sum_{k \neq j, k \neq i} U_{3}\left(r_{i}, r_{j}, r_{k}\right) .
$$

"This work was supported by the Polish grant RRI.14 
For SW potential:

$$
\begin{gathered}
U_{2 S T}=\left\{\begin{array}{lr}
\epsilon A\left[B\left(\frac{r_{i j}}{\sigma}\right)^{-4}-1\right] \times \exp \left[\left(\frac{r_{i j}}{\sigma}-a\right)^{-1}\right], & \text { if } \frac{r_{i j}}{\sigma}<a ; \\
0, & \text { if } \frac{r_{i j}}{\sigma} \geq a
\end{array}\right. \\
U_{3 S W}=h\left(r_{i j}, r_{i k}, \Theta_{i}\right)+h\left(r_{i j}, r_{j k}, \Theta_{j}\right)+h\left(r_{i k}, r_{j k}, \Theta_{k}\right) .
\end{gathered}
$$

If $\frac{r_{i j}}{\sigma}<a$ and $\frac{r_{i k}}{\sigma}<a$

$$
h\left(r_{i j}, r_{i k}, \Theta_{i}\right)=\varepsilon \lambda \exp \left[\gamma\left(\frac{r_{i j}}{\sigma}-a\right)^{-1}+\gamma\left(\frac{r_{i k}}{\sigma}-a\right)^{-1}\right] \times\left(\cos \Theta_{i}+\frac{1}{3}\right)^{2},
$$

otherwise $h\left(r_{i j}, r_{i k}, \Theta_{i}\right)=0$.

The AT potentials have form:

$$
\begin{gathered}
U_{2 A T}=\varepsilon\left[\left(\frac{r_{i j}}{\sigma}\right)^{12}-2\left(\frac{r_{i j}}{\sigma}\right)^{6}\right], \\
U_{3 A T}=Z \cdot \frac{1+3 \cos \Theta_{i} \cos \Theta_{j} \cos \Theta_{k}}{\left(r_{i j} r_{i k} r_{j k}\right)^{3}},
\end{gathered}
$$

where $\Theta_{i}, \Theta_{j}, \Theta_{k}$ and $r_{i j}, r_{i k}, r_{j k}$ represent the angles and sides of the triangle formed by the three atoms $i, j$, and $k$. There are seven parameters in SW potential $(A, B, \varepsilon, \sigma, a, \lambda, \gamma)$. Set of parameters: $A=7.049556277, B=0.6022245584$, $a=1.8, \lambda=21.0, \gamma=1.20, \sigma=2.0951 \AA, \varepsilon=2.17 \mathrm{eV}$, proposed in [1] is used.

For AT model only three parameters are required $(\varepsilon, \sigma, Z)$. Following [2] $\sigma=2.2951 \AA, \varepsilon=2.817 \mathrm{eV}, \mathrm{Z}=3484.0 \mathrm{eV} \AA^{9}$.

Our simulations were carried out using 10 layers of $8 \times 8 \mathrm{Si}$ atoms. Two bottom layers were fixed during simulations. The remaining atoms had initial velocities corresponding to a required temperature. The periodic boundary conditions in [110] and [110] directions were applied. Standard NVT (constant number of particles, constant volume and constant temperature) technique was used with velocities scaling at any iteration step.

If we use the ideal diamond structure as an initial configuration (without any dimerization) the final surface reconstruction is a random mixture of the $c(2 \times 2)$ and the $(2 \times 1)$ reconstructions. The final configurations depend on initial velocities directions. It is a metastable state. In order to find the lowest energy configuration we had to start from pure the $c(2 \times 2)$ or the $(2 \times 1)$ reconstructions. After MD iterations we got the reconstruction energy (relative to the diamond structure),

\begin{tabular}{|c|c|c|c|c|}
\hline 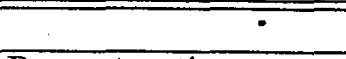 & $\overline{\mathrm{SW}(2 \times 1)}$ & $\overline{\mathrm{AT}(2 \times 1)}$ & SW c $(2 \times 2)$ & $\overline{\mathrm{AT} \mathrm{c}(2 \times 2)}$ \\
\hline $\begin{array}{l}\text { Reconstruction energy } \\
\text { (eV/surface atom) }\end{array}$ & 0.84 & 0.74 & 0.77 & 0.90 \\
\hline Bond length $(\AA)$ & 2.41 & 2.34 & 2.47 & 2.32 \\
\hline $\begin{array}{l}\text { First layer inward } \\
\text { relaxation }(\AA)\end{array}$ & 0.096 & 0.31 & 0.10 & 0.35 \\
\hline
\end{tabular}
dimer length and layers relaxations. The results are summarized in Table I. The experimental value of dimer length is about $2.4 \AA$.

TABLE I.

Si (001) surface reconstruction 
For Stillinger-Weber potential at low temperatures the $(2 \times 1)$ dimer reconstruction is about $0.07 \mathrm{eV}$ per surface atom more preferable than the $c(2 \times 2)$ structure which is in agreement with the experimental reconstruction observed by STM [8]. Contrary, for Axilrod-Teller type potential $c(2 \times 2)$ structure is lower by $0.16 \mathrm{eV}$ than $(2 \times 1)$ structure. The results of our MD studies of $(2 \times 1)$ reconstruction for SW potential agree perfectly with published results $[10,11]$.

Silicon surface is stable, at $1500 \mathrm{~K} \mathrm{SW}$ potential gives the same average value of dimer length, all the dimers remain unbroken but some of them are tilted.

The next problem analyzed here are the surface defects. In [9] Hammers and Köhler proposed, on the basic of their STM studies, three types of defects: $A-$ single dimer vacancy, $B$ - double dimer vacancy, $C-$ two neighbouring atoms (not forming a dimer) vacancy.

In addition we studied: $D$ - dimer vacancy (like $A$ ) with lower atom dimerization - the Pandey defect, $E$ - single atom vacancy. These defects are shown in Fig.1.

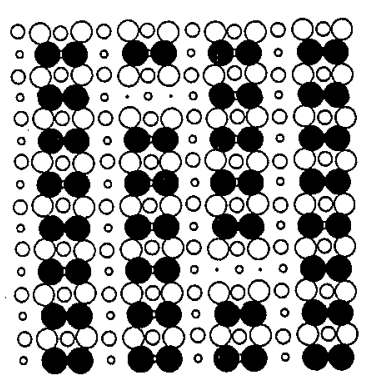

A

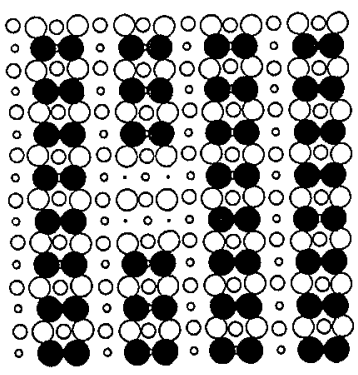

$\mathrm{B}$

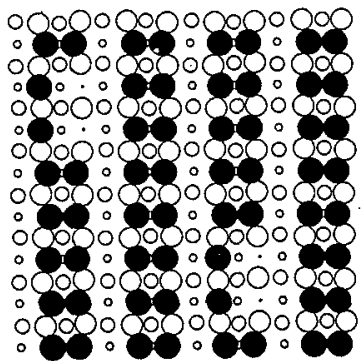

C

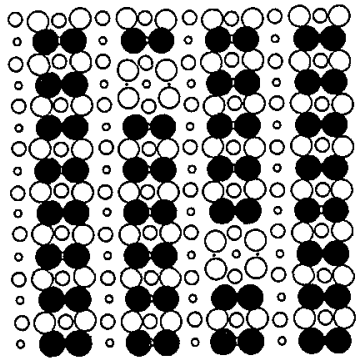

$\mathrm{D}$



E

Fig.1. Various kinds of defects $(A, B, C, D, E$ as described in text)

We carried out MD simulation in order to estimate the relative energies of various defects. We used similar geometry as in part $A$. In order to enable direct energetic comparisons the surface layer composes always of 60 atoms (e.g., one $D$ defect or four $E$ defects). The simulations were carried out at $30 \mathrm{~K}$ and $300 \mathrm{~K}$. Results are summarized in Table II. When analyzing Table II it should 
be remembered that SW potential is very short ranged (cutoff at next nearest neighbour distance) and the major contribution to energy comes from dimers and nearest neighbours. The number of dimers for $D$ is higher then for $A$ and $B$, which in turn is higher than for $C$ and $E$. At $300 \mathrm{~K}$ the situation is slightly different because the thermal energy is sufficient for lower layer dimerization.

TABLE II.

Energies ( $\mathrm{eV}$ per atom vacancy) of various defects relative to $\mathrm{D}$ defect

\begin{tabular}{l|c|c|c|c|c|c}
\hline \hline potential & temp. $[K]$ & A & B & C & D & E \\
\hline SW & 30 & 0.60 & 0.61 & 1.43 & 0 & 1.52 \\
SW & 300 & 0.08 & 0.06 & 0.93 & 0 & 1.08 \\
AT & $\mathbf{3 0}$ & 1.03 & 0.02 & 0.70 & 0 & 0.03
\end{tabular}

In summary the SW potential despite its simplicity gives good description of the Si (001) surface reconstruction. The AT potential seems to be less satisfactory for the clean surface (prefers the $c(2 \times 2)$ over the $c(2 \times 1)$ configuration). Moreover, for the AT potential at room temperature the surface defects make the surface unstable.

However, as will be published elsewhere [12], for the description of steps the AT potential is much better than the SW potential.

\section{References}

[1] F. Stillinger, T. Weber, Phys. Rev. B 31, 5262 (1985).

[2] E. Pearson, T. Takai, T. Halicoglu, W.A. Tiller, J. Cryst. Growth 70, 33 (1984).

[3] J. Tersoff, Phys. Rev. Lett. 56, 632 (1986).

[4] B.W. Dodson, Phys. Rev. B 35, 2795 (1987).

[5] K.E. Khor, S. Das Sarma, Phys. Rev. B 38, 3318 (1988).

[6] M.I. Baskes, J.S. Nelson, A.F. Wright, Phys. Rev. B 40, 6085 (1989).

[7] D.K. Choi, T. Takai, S. Erkoc, T. Halicioglu, W.A. Tiller, J. Cryst. Growth 85, 9 (1987).

[8] R.J. Hammers, R.M. Tromp, J.E. Demuth, Phys. Rev. B 34, 5343 (1986).

[9] R.J. Hammers, V.K. Köhler, J. Vac. Sci. Technol. A 7, 2854 (1989).

[10] F.E. Abraham, I.P. Batra, Surf. Sci. 163, L752 (1985).

[11] K.E. Khor, S. Das Sarma, Phys. Rev. B 36, 7733 (1987).

[12] J.M. Holender, C. Jedrzejek, to be published. 